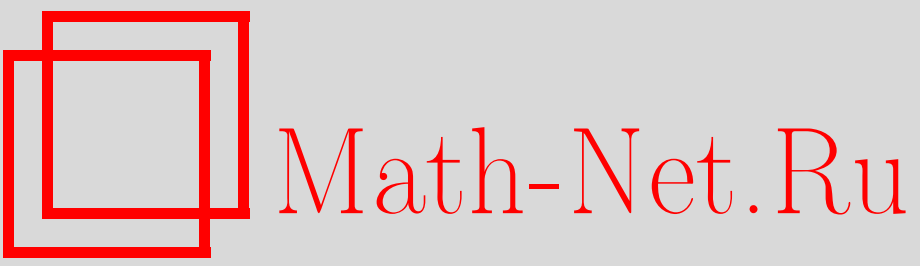

К. М. Феоктистов, Расчет верхних и нижних цен платежных обязательств посредством пополнения рынка, УМН, 1998, том 53, выпуск 2, 165-166

DOI: https://doi.org/10.4213/rm35

Использование Общероссийского математического портала Math-Net.Ru подразумевает, что вы прочитали и согласны с пользовательским соглашением

http://www.mathnet.ru/rus/agreement

Параметры загрузки:

IP: 54.80 .97 .219

26 апреля 2023 г., 13:53:12 


\title{
РАСЧЕТ ВЕРХНИХ И НИЖНИХ ЦЕН ПЛАТЕЖНЫХ ОБЯЗАТЕЛЬСТВ ПОСРЕДСТВОМ ПОПОЛНЕНИЯ РЫНКА
}

\author{
К. М. ФЕОКТИСТОВ
}

В работе изучается вопрос расчета цен платежных обязательств Европейского типа на конечном полиномиальном рынке, обобщающем биномиалную модель рынка Кокса, Росса и Рубинштейна. Хорошо известно (см. [1]) соотношение, связываюшее безарбитражные цены покупки и продажи обязательств Европейского типа:

$$
\mathbb{C}_{*}=\sup _{\pi \in \Pi_{*}} X_{0}^{\pi}=\inf _{p \in \mathbb{P}^{*}} \mathrm{E}_{p} \frac{F_{T}}{S_{T}^{0}} \leqslant \sup _{p \in \mathbb{P}^{*}} \mathrm{E}_{p} \frac{F_{T}}{S_{T}^{0}}=\inf _{\pi \in \Pi^{*}} X_{0}^{\pi}=\mathbb{C}^{*}
$$

где: $\Pi_{*}=\left\{\pi: \pi\right.$ - самофинансируемая стратегия, $\left.X_{T}^{\pi} \leqslant F_{T}\right\}, \Pi^{*}=\{\pi: \pi$ - самофинансируемая стратегия, $\left.X_{T}^{\pi} \geqslant F_{T}\right\}, F_{T}$ - обязательство Европейского типа, $S_{t}^{0}$ - безрисковьй актив, $\mathbb{P}^{*}$ - множество мартингалшных мер, эквивалентных исходной.

Заметим, что в случае полного рынка верхняя и нижняя цены образуют одну, "справедливую”, цену, которая находится по формуле: $\mathbb{C}=\mathrm{E}_{p^{*}} \frac{F_{T}}{S_{T}^{0}}$, где $p^{*}$ - единственная мартингальная мера. Возникает естественная идея - пополнить исходный рынок дополнительньми активами до полного, а затем вычислить справедливую цену (зависящую от способа пополнения рынка).

На исходном вероятностном пространстве (имеющем структуру полиномиального дерева) задан $(K+1)$-мерньй случайньй процесс цен $S_{t}=\left(S_{t}^{0}, \ldots, S_{t}^{K}\right)$, где $S^{0}$ - безрисковый актив $\left(S_{t+1}^{0} \stackrel{\text { def }}{=} S_{t+1}^{0}\left(A_{1}\right)=\cdots=S_{t+1}^{0}\left(A_{n}\right)\right)$, а $S^{1}, \ldots, S^{K}$ - рисковые. Рассматривается сужение этого процесса на редуцированное вероятностное пространство $\left(A, \mathscr{A}_{A}, P_{A}\right)$, описьвающее один узел $(t, A)$ полиномиального дерева (все обозначения взяты из [2]); $\mathscr{A}_{A}=\sigma\left\{A_{1}, \ldots, A_{n}\right\}$, где $A_{1}, \ldots, A_{n}$ - все вероятностные исходы, которые могут произойти в момент $t+1$ с положительной вероятностью. Число $n$ назьвается индексом ветвления (splitting index). Из рассмотрения исключается вырожденный случай наличия на рынке активов, линейно выражаемых через другие, поскольку они не влияют на построение хеджирующей стратегии. В [2] формулируются следующие критерии

КРИТЕРИЙ БЕЗАРБИТРАЖНОСТИ. РЫнок Полон $\Longleftrightarrow$

$$
(\underbrace{S_{t+1}^{0}, \ldots, S_{t+1}^{0}}_{K+1})^{T} \in \operatorname{ri}\left\{\operatorname{conv}\left\{S_{t+1}\left(A_{i}\right): i \in\{1 \ldots n\}\right\}\right\} .
$$

КРИТЕРИй пОлноты. Безарбитражный рынок полон $\Longleftrightarrow$

$$
\operatorname{dim}\left\{\operatorname{span}\left\{S_{t+1}\left(A_{i}\right): i \in\{1 \ldots n\}\right\}\right\}=n,
$$

где $S_{t+1}\left(A_{i}\right)=\left(S_{t+1}^{0}\left(A_{i}\right), \ldots, S_{t+1}^{K}\left(A_{i}\right)\right)^{T}$ - вектор стоимостей активов.

Используя утверждения вьпуклой алгебры (см. [3]), можно получить следующий, эквивалентный, критерий: рынок полон и безарбитражен $\Longleftrightarrow$ система

$$
\left\{\begin{aligned}
\mathbf{S}_{t+1}(A) \cdot & =\underbrace{\left(S_{t+1}^{0}, \ldots, S_{t+1}^{0}\right)^{T}}_{K+1} \\
p_{i} & >0: i \in\{1, \ldots, n\}
\end{aligned}\right.
$$

имеет решение, и только одно.

Здесь: $\mathbf{S}_{t+1}(A)=\left(S_{t+1}\left(A_{1}\right), \ldots, S_{t+1}\left(A_{n}\right)\right)$ - матрица, описьвающая исходньй рынок, $p=$ $\left(p_{1}, \ldots, p_{n}\right)^{T}$ - вектор вероятностей событий $A_{1}, \ldots, A_{n}$. 
Задача минимального пополнения рынка: ввести в рассмотрение минимальное количество дополнительных активов $S^{K+1}, \ldots, S^{K^{\prime}}$, чтобы новьй рынок $\mathbf{S}^{\prime}$ был уже полон. Поскольку критерий неполноты безарбитражного рынка имеет вид:

$$
\operatorname{dim}\left\{\operatorname{span}\left\{S_{t+1}\left(A_{i}\right): i \in\{1, \ldots, n\}\right\}\right\}<n,
$$

то рынок должен пополняться зависимыми активами (увеличивая левую часть на единицу, не зависимьй функционалшно актив увеличивает и число вероятностных исходов - правую часть, сохраняя неравенство (3) и неполноту рынка).

КРИТЕРИЙ МИНИМАЛЬНОГО ПОПОЛНЕНИЯ РЫНКА. 1. $K^{\prime}=n$.

$$
\begin{aligned}
& \text { 2. }\left\{\begin{aligned}
S_{t}^{K+1}=f_{t}^{K+1}\left(S_{t}^{0}, \ldots, S_{t}^{K}\right) & \text { (система уравнений } \\
\ldots \ldots \ldots \ldots \ldots \ldots \ldots \ldots \ldots, & \text { и неравенств имеет } \\
S_{t}^{K^{\prime}}=f_{t}^{K^{\prime}}\left(S_{t}^{0}, \ldots, S_{t}^{K}\right) & \text { единственное решение })
\end{aligned}\right. \\
& \text { 3. }\left\{\begin{array}{cl}
\mathbf{S}_{t+1}(A) \cdot p=(\underbrace{S_{t+1}^{0}, \ldots, S_{t+1}^{0}}_{K+1})^{T} & \begin{array}{ll}
\text { (система уравнений } \\
\text { и неравенств имеет } \\
\text { единственное решение })
\end{array} \\
p_{i}>0: i \in\{1, \ldots, n\} &
\end{array}\right.
\end{aligned}
$$

Основным резултатом работы является следующая теорема:

ТЕОРЕМА (О ПОПОЛНЕНИИ РЫНКА).

$$
\mathbb{C}_{*}=\inf _{n \in \Pi} \mathbb{C}^{n} \leqslant \sup _{n \in \Pi} \mathbb{C}^{n}=\mathbb{C}^{*}
$$

где П - множество минимальных пополнений рынка, $\mathbb{C}^{n}-$ справедливая цена на пополненном рынке.

Идея доказательства заключается в построении взаимнооднозначного соответствия между П и $\mathbb{P}^{*}$, из которого в силу (1) сразу вытекает (5).

ЗАмечАниЕ. Все изложенные утверждения были сформулированы для одного узла полиномиального дерева (на редуцированном пространстве). Однако с помощью метода “обратной индукции" изложенные утверждения можно распространить и на весь полиномиальный рынок.

Работа выполнена под руководством А. В. Мелњникова, которому автор выражает свою благодарность.

\section{СПИСОК ЛИТЕРАТУРЫ}

[1] Мельников А.В. Финансовые рынки, стохастический анализ и расчет производных ценных бумаг. Москва: ТВП, 1997. [2] Taqqu M. and Willinger W. The analysis of finite security markets using martingales // Adv. Appl. Probab. 1987. V. 19. Р. 1-25. [3] Рокафоллар Р.Т. Выпукльй анализ. М.: Мир, 1973.

Московский государственньй

Принято редколлегией университет им. М. В. Ломоносова 22.01 .1998 